\title{
LE DROIT INTERNATIONAL NEOLIBERAL: LES CONTRIBUTIONS DU CONTENU ET DES SOURCES DU DI AU NEOCOLONIALISME
}

\author{
NEOLIBERAL INTERNATIONAL LAW: THE CONTRIBUTIONS OF IL'S CONTENT AND \\ SOURCES TO NEOCOLONIALISM
}
O DIREITO INTERNACIONAL NEOLIBERAL: AS CONTRIBUIÇÕES DO CONTEÚDO E DAS FONTES DO DI AO NEOCOLONIALISMO

\author{
Felipe Costa Lima ${ }^{1}$
}

\begin{abstract}
Résumé
La fin de la Seconde Guerre Mondiale et l'établissement de l'ONU ont provoqué des nouveaux changements dans les relations mondiales. Non seulement la décolonisation est devenue un des objectifs rhétoriques primordiaux de la société internationale, mais également, le processus de décolonisation ultérieur a aussi proportionné un changement de la composition de cette société (le système de l'ONU composé avec une majorité des pays sous-développés). De ce fait, il semblait que la société internationale et le droit international vivraient une nouvelle ère de développement mutuel. Toutefois, des nouvelles discordes ont éclaté au sein de la société internationale, plutôt par rapport au contexte économique. L'objectif le plus fondamental de cet article est la démonstration de la continuation de l'utilisation du droit international (son contenu et ses sources) comme un facteur légitimant l'impérialisme des puissances globales sur les pays de la " périphérie » du monde, particulièrement à partir de la Pax Americana (l'hégémonie néolibérale). Afin d'atteindre cet objectif crucial, les méthodologies de la Théorie Critique et de l'Économie Politique International (EPI) se révèlent fondamentales, ainsi que la relation étroite entre les domaines de connaissance du Droit International et des Relations Internationales. Le résultat le plus fondamental de cette recherche met en lumière des politiques néocoloniales en arrière-plan du DI.
\end{abstract}

Mots-clés: Néocolonialisme; Néolibéralisme; Droit International; Hégémonie; Gouvernance Économique Internationale.

\begin{abstract}
The end of the Second World War and the establishment of the UN have provoked new changes as regards international relations. Not only has decolonization became one of the most fundamental rhetorical objectives of international society, but also entrenched changes towards the composition of this society (a majority of underdeveloped countries in the UN system). As a consequence, it seemed that international society and international law would propitiate a new era of mutual development. Nonetheless, new discords have erupted in international society, mainly as concerns the economic realm. The main objective of this article is the demonstration of the continued use of international law (its content and sources) as a legitimizing aspect of global powers imperialism over peripherical States of the world, primarily since the Pax Americana (neoliberal
\end{abstract}

\footnotetext{
${ }^{1}$ Doutorando em Direito Internacional na Université de Strasbourg, França, e Doutorando em Relações Internacionais na Pontifícia Universidade Católica de Minas Gerais (PUC-Minas). ORCID: https://orcid.org/0000-0001-6390-3950. E-mail: felipecostalimas@gmail.com.
} 
hegemony). To achieve this crucial goal, Critical Theory and International Political Economy's (IPE) methodologies will be essential, as well as the intrinsic relation between International Law and International Relations' scopes of knowledge. The utmost result of this research highlights neo-colonial policies in DI's background.

Keywords: Neocolonialism; Neoliberalism; International Law; Hegemony; International Economic Governance.

\section{Resumo}

X O fim da Segunda Guerra Mundial e o estabelecimento da ONU provocaram novas mudanças nas relações internacionais. Não somente a descolonização se tornou um dos objetivos retóricos primordiais da sociedade internacional, mas também o posterior processo de descolonização proporcionou uma mudança da composição dessa sociedade (o sistema da ONU tornou-se composto majoritariamente de países subdesenvolvidos). Desse modo, parecia que a sociedade internacional e o direito internacional viveriam uma nova era de desenvolvimento mútuo. Não obstante, novas discórdias apareceram no seio da sociedade internacional, sobretudo no que concerne ao contexto econômico. Objetivo fundamental deste artigo é a demonstração Da continuação da utilização do direito internacional (seu conteúdo e suas fontes) como um fator legitimador ao imperialismo das potências globais sobre os países periféricos, particularmente a partir da Pax Americana (a hegemonia neoliberal). A fim de alcançar esse objetivo crucial, as metodologias da Teoria Crítica e da Economia Política Internacional (EPI) serão fundamentais, assim como a estreita relação entre os domínios do Direito Internacional e das Relações Internacionais. O resultado mais importante dessa pesquisa parece demonstrar políticas neocoloniais escondidas por trás do DI.

Palavras-chave: Neocolonialismo; Neoliberalismo; Direito Internacional; Hegemonia; Economia Política Internacional.

\section{INTRODUCTION}

D’abord, nous mettrons en évidence la méthodologie la plus essentielle utilisée dans cet article, c'est-à-dire, la théorie critique du droit et des relations internationales. La théorie critique rétablit le lien avec la grande tradition philosophique, notamment hégélienne, face à la rationalisation envahissante des sciences sociales, mettant en place une sorte de controverse permanente avec le positivisme et sa « rationalité en soi », étant donné que, au nom de l'imposition de la rationalité, ce type de science établi un type de domination politique déterminée et nonavouée (BIANCHI, 2016; BUTLER, 2011; CARTY, 1991; HIRSHC, 1975; KOSKENIEMI, 2008; MARKS, 2008; ORFORD, 2003).

Dans ce contexte, ce type d'approche tend à risquer de maintenir des pratiques sociales qui reproduisent seulement les formes dominantes de l'activité sociale, ces dernières établissant un caractère illégitime à n'importe quelles autres formes de compréhension de la " réalité " (POLANYI, 2000). Par conséquent, la naturalisation de la réalité et la méconnaissance de la 
détermination sociale de ces théories imposent une soumission sans réserve à la domination. Cela s'applique à la maxime sur laquelle « la connaissance c'est le pouvoir ». Enfin, les théories positivistes établissaient des caractéristiques dépersonnalisées et idéologiques à la " réalité " (CARTY., 1991; MARKS, 2008).

Afin de nous concentrer sur les prémisses de la théorie critique de l'École de Francfort, il est opportun de souligner l'importance des héritages conceptuels marxiens qui ont influencé cette dernière. Entre autres, la critique de l'idéalisme constitue une des plus fondamentales contributions de Marx et Engels pour l'appréhension de concepts significatifs de la modernité (MARKS, 2008), surtout le processus d'aliénation provoqué par l'idéalisme (KOSKENIEMI, 2008). À cet égard, dans l'ouvrage I'Idéologie allemande, Marx et Engels (1999) mettent en relief l'objectif des classes dominantes de transformer leurs intérêts particuliers en des intérêts universels.

De surcroit, la conception matérialiste a été une conséquence et aussi une cause de la critique de l'idéalisme. S'agissant de ce concept, I'histoire doit être comprise en termes matérialistes (MARX, 2011), étant donné que les modes de production spécifiques d'une période historique et des relations de production associées sont cruciales pour la compréhension du développement humain.

Encore par rapport à cette conception, Antonio Gramsci (1982) soutient que, d'abord, les processus de production ne sont pas isolés de la réalité, puisqu'ils sont historiquement construits et déterminés socialement. Par conséquent, les développements de la structure et de la superstructure se produisent réciproquement à travers la relation dialectique des facteurs économiques et socioculturels, respectifs. De ceci, l'ensemble complexe, contradictoire et discordant de la superstructure est le reflet des relations sociales de production (MORTON, 2007).

De ce fait, le passage d'une structure à une superstructure intervient à partir du moment où une idéologie particulière devient universelle et hégémonique. Cependant, même si elle atteint I'hégémonie, les relations dialectiques ne cessent jamais, de sorte que les forces pros et contrehégémoniques influencent continuellement la conformation de la structure et de la superstructure, dans une construction sociale constante (GRAMSCI, 1982). La superstructure a une certaine autonomie par rapport à la structure, puisque c'est au niveau idéologique que les hommes prennent conscience du monde économique. Ainsi, « il est possible de comprendre théoriquement comment les relations sociales régressives et exploratoires de production continuent à persister en raison d'idéologies favorables » (MORTON, 2007, p. 96, notre traduction)2.

La dialectique marxienne se révèle aussi un des héritages les plus essentiels, car celle-ci fournit une perspective de changement constant dans les relations humaines. Concernant le

\footnotetext{
2 "Possible to theoretically understand how regressive or exploitative social relations of production may still persist because of supportive ideologies".
} 
concept susmentionné, celui-ci suppose une vision globale du réel, afin de clarifier les éléments sociaux distincts comme liés à la même réalité. Cette méthode permet la révolution du status quo, en vertu de la compréhension du monde comme le résultat de la praxis humaine (CARTY, 2008; KOSKENIEMI, 2008; MARKS, 2008).

Enfin, le dernier concept à être appréhendé est la vision de totalité marxienne qui est centrale pour comprendre les dimensions globales du capitalisme et, par conséquent, formuler une critique de l'impérialisme. Ce concept propose que les productions matérielles et intellectuelles des nations individuelles deviennent universelles, en imposant l'interdépendance économique comme un facteur immuable. Au nom de cela, la considération du monde dans son ensemble comme une dynamique entière semble indispensable, la méconnaissance de ladite dynamique pouvant en effet instaurer une compréhension fragmentée et fétichiste de la réalité (MARKS, 2008).

Lesdits héritages introduits ci-avant sont les bases fondamentales du projet de la théorie critique. À ce propos, cette conception cherche à surmonter la conscience réifiée par rapport aux idéaux grandiloquents de la société bourgeoise et du projet libéral (la liberté, la justice, l'égalité, les droits de l'homme) (MARKS, 2008). De plus, cette théorie est caractérisée par une certaine complexité, dans la mesure où elle est directement liée au temps et à l'espace, afin de transcender les caractéristiques de l'ordre existant et de problématiser les processus de changement dans plusieurs sphères, telles que les relations sociales, le pouvoir et les institutions (COX, 1981; COX; SCHECHTER, 2002).

À partir de ces héritages, cette école de pensée met en évidence trois propositions cruciales qui permettent de fonder l'analyse du développement du droit international : l'impossibilité de séparation de l'objet du sujet, la contestation du status quo et la recherche d'une conception subjective/historique de la vérité (BUTLER, 2011). D'après ces arguments, nous pouvons mettre l'accent sur le domaine de l'Économie Politique International (EPI), lequel émerge à partir des années 1970 en vertu de la croissance du phénomène d'interdépendance des États.

Vis-à-vis de l'EPI, nous pouvons affirmer que cette méthode d'analyse est aussi basée sur les héritages marxiens. Une des thématiques centrales des auteurs d'inspirations marxiennes qui étudient les relations internationales est le dépassement de la scission entre l'économie et la politique. En ce sens, vu l'importance des concepts de domination et hégémonie, il s'avère presque impossible de les comprendre sans mettre l'accent sur ses causes et conséquences aux niveaux nationaux et internationaux. De ce fait, la reproduction des relations sociales capitalistes et le processus d'exploitation présupposent la séparation formelle entre la politique et l'économie, de manière à « cacher » ses profondes interdépendances (VIGEVANI et al., 2011).

En opposition à ces théories dominantes, Vigevani (2011) met en relief que la contribution du marxisme réside dans l'introduction de la question économique en tant que facteur explicatif 
non seulement des relations de pouvoir, mais aussi des relations entre les États du système international, afin de comprendre la structure et la dynamique de celui-ci. Enfin, il existe une intense relation d'interaction entre les sphères économiques et politiques ; ainsi, seulement une réflexion qui n'exclut pas la variable économique pour l'étude de la politique, et vice versa, est capable de comprendre le système international dans son ensemble.

Il est important d'affirmer que cette étude de l'interaction réciproque et dynamique dans les relations internationales entre l'accumulation de la richesse et la poursuite de la puissance est allée au-delà du marxisme. Toutefois, quoique nous reconnaissions l'importance des courants théoriques orthodoxes réalistes, surtout Stephan Krasner (1976) et Robert Gilpin (1975, 2001), ainsi que libérales, plus particulièrement Joseph Nye et Robert Keohane (2001), nous utiliserons notamment dans cet article le courant théorique marxiste susmentionné et la nouvelle économie politique internationale (NEPI). À propos de la NEPI, elle est née en réaction à l'école orthodoxe en relations internationales, c'est-à-dire, l'école néoréaliste et l'institutionnalisme néolibérale (synthèse néo-néo). Dans ce contexte, à partir d'une approche hétérodoxe ainsi que profondément multidisciplinaire (à savoir science politique, économie, sociologie, anthropologie, droit, histoire), la NEPI met l'accent sur d'autres contextes essentiels, en particulier l'émergence d'une diplomatie économique, les changements de la géographie globale et les finances mondiales (PAQUIN, 2009).

En outre, il s'avère indispensable de mettre en évidence deux grands acteurs de cette théorie hétérodoxe, à savoir Susan Strange $(1996,1997)$ et Robert Cox. Alors que le premier adopte une approche réaliste non centré sur les États mais sur l'ensemble des acteurs internationaux, le second adopte une ligne de pensée néogramscienne, c'est-à-dire liée aux classes dominantes et à la façon dont elle acquiert son pouvoir hégémonique (PAQUIN, 2009).

Enfin, cette introduction méthodologie se traduit comme indispensable pour la compréhension des processus extrêmement complexes de la réalité internationale, spécialement les relations entre le droit international et la structure néolibérale (néocolonialisme), étant donné que, à partir d'une relation dialectique, le premier semble fonctionner comme une « loi éternelle » afin de mettre en place les intérêts du second.

Ainsi, dans cette étude, nous mettrons en évidence les relations dialectiques entre le droit international contemporain et le projet néolibéral de développement, notamment à partir des années 1970. C'est pourquoi, d'abord, nous analyserons les relations dialectiques entre le contenu du droit international contemporain et l'impérialisme (2). Par la suite, nous pourrons envisager les relations dialectiques entre les sources du droit international contemporain et l'impérialisme (3). Donc, il semble possible d'envisager des relations néocolonialistes entre les grandes puissances globales et les pays périphériques, en utilisant toujours le droit international comme une source de justification de l'impérialisme. Ainsi l'objectif le plus fondamental de cet article constitue en la 
démonstration de la continuité des caractéristiques impérialistes du droit international en arrièreplan, à partir des profonds changements dudit droit, notamment dans les contextes de l'institutionnalisation d'une gouvernance économique internationale. Dans ce contexte, le droit international économique d'aujourd'hui semble constituer un des mécanismes impérialistes les plus importants, surtout depuis les années 1970, étant donné l'expansion capitaliste à la majorité des pays du monde.

\section{LE CONTENU DU DROIT INTERNATIONAL CONTEMPORAIN ET L'IMPERIALISME}

Afin d'expliciter le caractère potentiellement impérialiste du contenu du droit international contemporain, nous étudierons, d'abord, les origines du néolibéralisme et son expansion à l'échelle mondiale (2.1). Ensuite, nous mettrons en évidence le rôle de la Gouvernance Économique Mondiale dans l'application du projet néolibéral (2.2), surtout du FMI (2.2.1), de la BM (2.2.2) et du GATT/OMC (2.2.3).

\subsection{LES ORIGINES DU NEOLIBERALISME ET SON EXPANSION A L'ECHELLE MONDIALE}

Selon Arrighi et Silver (2003), au regard de la Pax Americana, I'après-Seconde Guerre mondiale a fourni la «Belle Époque » de l'hégémonie américaine, qui était basée sur le « développementalisme ", c'est-à-dire, l'alliance capital-travail et la grande puissance hégémonique (les États-Unis). II est opportun de mettre en évidence que cette hégémonie a été relativement restreinte aux pays capitalistes et à une grande partie des pays non-alignés depuis la Seconde Guerre Mondiale jusqu'à la chute de la URSS ; depuis ce dernier événement, I'hégémonie susmentionnée a été étendue à l'échelle mondiale, surtout aux années 1990 et 2000.

Ce processus change cependant dans les décennies suivantes, compte tenu de la crise hégémonique américaine profonde, en particulier dans les années 1970 et 1980. Premièrement, l'augmentation de la compétitivité mondiale, fournie par le processus de rattrapage de certains États du centre capitaliste (I'Allemagne et le Japon, par exemple), a créé une crise rentable dans les industries américaines. Par ailleurs, le confinement des difficultés du communisme et du nationalisme (révolution iranienne de 1979, la guerre du Vietnam), l'augmentation des prix du pétrole et l'invasion de l'URSS en Afghanistan ont alimenté une crise de légitimité dans le leadership des États-Unis (ARRIGHI; SILVER, 2003).

En ce sens, des changements radicaux ont eu lieu dans les politiques de ce pays, en particulier dans le contexte de l'économie, à travers une politique monétaire contra cycliste, la 
hausse des taux d'intérêt, les réductions des impôts pour les riches, la liberté presque sans restriction pour les entreprises capitalistes ainsi que la dévaluation du dollar, laquelle a abouti à I'intensification de la compétition pour le capital (des politiques néolibérales). Grâce à ces politiques, les États-Unis deviennent un entrepôt financier mondial, car cet État s'est transformé en le plus grand bénéficiaire des investissements étrangers au monde (ARRIGHI; SILVER, 2003).

Il est perçu que le mouvement pro-marché est né d'une perspective nationale (États-Unis), qui s'est ensuite étendue au contexte mondial afin de profiter à ce pays (ARRIGHI; SILVER, 2003; MORTON, 2007; PIJL, 2005; RUPERT, 2007). II semble que les États-Unis étaient le principal articulateur et bénéficiaire de l'imposition du néolibéralisme dans le monde, tout comme le Royaume-Uni l'était pendant I'hégémonie libérale. Selon Cox (COX, 1981; COX; SCHECHTER, 2002), l'empire américain a pour modus operandi la tentative de contrôler les États, à travers la structuration de son système économique dans un vaste marché de capitaux, de biens et de services.

II est important de noter aussi que des coalitions transnationales d'intérêts existent dans divers domaines, tels que divers réseaux de directeurs de sociétés et de banques internationales, de groupes de planification et de médias internationaux. Ainsi, cette transformation d'intérêts spécifiques en intérêts universels découle de la planification d'intellectuels organiques, de politiciens professionnels et de forces culturelles et ethniques. La bourgeoisie n'a pas de dogmes stricts, agissant en fonction des obstacles que chaque situation historique impose (PIJL, 2005).

Ainsi, par la généralisation et l'universalisation d'une idéologie de marché, les valeurs se propagent et son expansion est justifiée comme bénéfique pour le monde entier. Toutefois, comment les États-Unis ont transformé ses intérêts particuliers en généraux ? Nous pouvons peutêtre trouver la réponse si nous analysons la soi-disant Gouvernance Économique Mondiale.

\subsection{LE ROLE DES ORGANISATIONS INTERNATIONALES DANS L'APPLICATION DU PROJET NEOLIBERAL}

L'Accord de Bretton Woods de 1944 constitue une série d'accords économiques adoptés par divers pays, visant à la stabilité du système monétaire international et à la recherche d'un nouveau système après l'effondrement de l'étalon or. Toute la période de négociation a été fortement marquée par la présence de deux personnalités tout à fait pertinentes pour la prise de décision et le processus de prise de décision des pays concernés. D'un côté, l'économiste britannique Keynes et, de l'autre côté, l'américain White ; à la fin, le plan White a été la base la plus primordiale pour l'établissement des Accords de Bretton Woods, lesquels sont considérés comme 
le piédestal économique du système international à partir de $1944^{3}$. La conséquence la plus directe de ces accords a été la création de certaines institutions qui jouent un rôle clé dans la nouvelle dynamique du nouveau système monétaire international, entre autres le Fond Monétaire International (FMI) ; la Banque Internationale pour la Reconstruction et le Développement (BIRD), qui deviendra plus tard la Banque mondiale (BM); et l'Accord Général sur les Tarifs Douaniers et le Commerce (GATT, en anglais), lequel deviendra plus tard I'Organisation Mondiale du Commerce (OMC) (OLIVEIRA; MAIA; MARIANO, 2008).

II semble que toutes les prémisses néolibérales déjà mentionnés dans cet article ont été adoptés par la Gouvernance Économique Mondiale, à travers les institutions publiques telles que la BM, le FMI, d'autres banques multilatérales de développement, ainsi que le GATT/OMC, sans oublier la majorité des gouvernements. Ces institutions sont définies comme étant toutes des institutions publiques, parce qu'elles devraient représenter les intérêts des citoyens du monde et promouvoir le bien public mondial (BUTLER, 2011; ORFORD, 2003). L'objectif de l'article à cette étape constitue en la démonstration non seulement du manque de neutralité de cette Gouvernance Économique Internationale et, en conséquence, du droit international économique actuel, mais encore de l'instrumentalisation de ces deux domaines aux intérêts hégémoniques des grandes puissances. II est indispensable de marquer la complexité de cette instrumentalisation aux intérêts hégémoniques, étant donné qu'il existe toujours des espaces de contestation contrehégémonique, même au sein desdites institutions. Néanmoins, il semble que l'hégémonie néolibérale américaine se révèle comme le principal bénéficiaire des politiques de ces organismes internationaux.

\subsubsection{Le FMI}

Le FMI et la BM ont été fondés en 1945, avec leurs bureaux principaux à Washington. Les objectifs de ces institutions ont été énoncés dans leurs statuts, entre autres, la promotion de la stabilité du système monétaire international et du libre-échange, ainsi que des taux de change stables et de la croissance économique (BM, 1989; FMI, 1944).

Particulièrement, quant au FMI, cette institution a comme but spécifique la promotion de la coopération monétaire à travers la consultation et la collaboration des membres de l'institution. Encore par rapport au FMI, rares sont les organisations internationales qui relient si clairement le poids effectif des différents États membres à leur influence sur le processus décisionnel (BUTLER, 2011). Après la réforme des quotas de l'organisation en 2010 , les dix quotas les plus grands se révèlent être les suivants : les États-Unis ont 17,68\% du total de quotas, le Japon a 6,56\%, la Chine

\footnotetext{
${ }^{3}$ Voir notamment sur cette question Oliveira, Maia et Mariano (2008).
} 
a $6,49 \%$, l'Allemagne a 5,67\%, la France a 4,29\%, le Royaume-Uni a 4,29\%, l'Italie a 3,21\%, l'Inde a 2,79\%, la Russie a 2,75\% et le Brésil a 2,35\% (FMI, 2018). Quoique les quotas puissent être révisés tous les cinq ans, les actions à droit de vote du FMI sont relativement rigides. De ce fait, les pays qui se développent sur le plan économique ont tendance à être sous-représentés, du fait que leur pouvoir de vote est à la traîne.

Par ailleurs, ce fond cherche l'établissement de la stabilité des taux de change, la mise en place d'un système multilatéral de paiements et de la réduction des restrictions de change (FMI, 1944, Art. 1). En outre, quant aux programmes de stabilisation, « le but d'un programme ou de conditions de prêt, selon le Fond, n'est pas de modifier la structure économique ou politique d'un membre, et donc les politiques sociales ou politiques d'un membre sont exclues de considération »(FMI, 1944, Art. 4, Section 3(b)).

\subsubsection{La BM}

Concernant la Banque Mondiale, cette institution a comme but spécifique le fournissement des prêts de projet pour la reconstruction et le développement dans les pays pauvres. Afin d'atteindre ce but, ladite institution cherche à faciliter l'investissement de capitaux à des fins productifs ; promouvoir l'investissement étranger privé et compléter l'investissement privé par des prêts sur son propre capital ; promouvoir la croissance équilibrée du commerce international et l'équilibre de la balance des paiements ; veiller à ce que des financements adéquats existent pour le « plus utile et urgent projet »; et tenir dûment compte des effets de ses options sur les conditions d'affaires parmi ses membres (BM, 1989, Art. 1). D'une façon similaire au FMI, le critère politique est aussi exclu par la BM (1989, Art. 4, Section 10).

Par rapport à la libéralisation du secteur financier, le système financier global positionne le secteur privé comme la principale source de capital pour la croissance (THOMAS, 2003). En particulier, jusqu'en 1999, le FMI cherchait activement à modifier sa Charte pour permettre à ses membres d'ouvrir leurs marchés financiers aux investisseurs étrangers (BUTLER, 2011).

\subsubsection{Le GATT/OMC}

Vis-à-vis du GATT/OMC, la libéralisation du commerce a été une caractéristique fondamentale afin d'intégrer l'économie mondiale depuis la fin de la Seconde Guerre Mondiale. Puis, selon Carolina Thomas (2003), les pays développés ont cherché à réduire les obstacles au commerce, à partir de la diminution de diverses opérations de réduction tarifaire. Parmi ces réductions, nous pouvons souligner celles des droits de douane, des contingents et d'autres 
mesures qui avaient précédemment empêché les mouvements transfrontières de marchandises. À partir de 1995, cet accord, qui comptait 122 membres, a été absorbé par l'OMC, c'est-à-dire, qu'un mouvement mondial vers la libéralisation des échanges s'est transformé en une institution au caractère mondial. Depuis lors, l'OMC a accru son pouvoir de renforcer les accords commerciaux existants et de poursuivre de nouvelles voies de libéralisation, telles que les flux d'investissement (BUTLER, 2011). Le libre-échange a également été encouragé au niveau régional, notamment par des institutions comme le NAFTA, le Mercosur et l'UE. Enfin, il paraît nécessaire dans cet article, compte tenu des arguments avancés ci-avant, d'affirmer que les institutions internationales et le droit international économique ne sont pas neutres ; au contraire, ceux-ci sont des sources de maintenance et de justification d'un type de progrès spécifique, qui bénéficie qu'à certains états, notamment aux États-Unis.

Nous pouvons envisager, à partir des buts de ces institutions que la soi-disant intégration économique mondiale est basée profondément dans le projet néolibéral, comme si celui-ci était le chemin le plus naturel et le plus universel vers la croissance économique et vers la sécurité de I'humanité toute entière (BUTLER, 2011). Compte tenu de cela, il est possible de soutenir que l'idéologie néolibérale promue autour du monde serait la seule approche appropriée aux problèmes de sécurité et de développement (THOMAS, 2003).

De surcroit, lorsque nous regardons la réalité internationale, cette conclusion trouve des bases plus solides. Le FMI et la BM ont été impliqués, en particulier depuis 1979, dans les programmes d'ajustement structurel dans le sud global. Lesdits programmes, surtout ceux du FMI, avaient comme objectifs l'établissement d'un environnement approprié dans les pays emprunteurs, à partir de la privatisation des services publics, de la libéralisation du commerce et des finances, ainsi que de la production et l'assouplissement des lois du travail et de l'environnement.

À titre d'exemple, le diagnostic donné par ces institutions à la crise de l'Amérique Latine des années 1980 et 1990 est en résonance avec les arguments susmentionnés. Selon l'avis de Petrônio Portela Filho (1994), les causes externes de la crise de cette région ont été négligées par ces institutions internationales, étant donné que le processus de « substitution des importations ${ }^{4}$ » avait déjà perdu son importance dans la plupart des pays de cette région lorsque la crise de la dette extérieure s'est produite. Par ailleurs, cet auteur affirme que les distorsions économiques qui ont le plus influencé la crise étaient liées au secteur financier, et non pas au modèle de développement de la région.

Encore vis-à-vis de cette région, les réformes défendues par l'idéologie du néolibéralisme, comme les privatisations du secteur public, les révisions des codes du travail et la profonde

\footnotetext{
${ }^{4}$ Sommairement, le processus d'industrialisation par substitution aux importations se fonde sur des barrières tarifaires ou non tarifaires permettant au pays de produire lui-même ce qu'il importe à l'origine, créant un développement autocentré.
} 
soutenance aux investissements étrangers ont eu des conséquences catastrophiques dans tous les contextes, notamment le social (la précarisation des services sociaux, la fin de subsides aux paysans), l'économie (des crises fiscales, de l'inflation, des déséquilibres budgétaires) et politique (des tensions de classes, l'affaiblissement des syndicats) (SILVA, 2012).

Ainsi, en raison des crises qui ont découlé de la dette extérieure et de la balance de paiements des pays du tiers monde, les États-Unis cherchaient un consensus sur l'adoption d'autres politiques des gouvernements qui pouvaient mettre en place le projet néolibéral, avec le soutien des lesdits « institutions internationales ».

II est intéressant de mettre en lumière que, pendant la période néolibérale, l'expansion du commerce mondial des marchandises a été deux fois plus fort que la croissance de la production mondiale. En effet, en 2005, la valeur des exportations mondiales de marchandises a atteint pour la première fois la barre des 10000 milliards de dollars. Cependant, malgré ce succès apparent, la profonde croissance de l'inégalité dans le monde et à l'intérieur des pays a augmenté (PNUD, 2006 in BUTLER, 2011).

Par exemple, encore selon Butler (2011), la grande adhésion des pays en développement aux politiques néolibérales préconisées par le Consensus de Washington a été associée non à une amélioration mais à une détérioration de la performance économique de ceux-ci, comme en témoigne la baisse du taux de croissance médian de leur revenu par habitant de 2,5\% en 1960-79 à $0 \%$ en 1980-98. De surcroit, Butler cite des autres données qui assoient cette affirmation :

Les $40 \%$ les plus pauvres de la population mondiale, soit 2,5 milliards de personnes vivant avec moins de 2 dollars par jour, représentent $5 \%$ du revenu mondial, tandis que les $10 \%$ les plus riches en représentent $54 \%$. (...) Bien qu'il existe des ressources techniques et non plus des obstacles logistiques à l'abolition de la pauvreté, plus de 800 millions de personnes souffrent de la faim et de la malnutrition. Un milliard de personnes n'ont pas accès à l'eau potable. Alors que le commerce peut être l'un des moteurs les plus efficaces de la croissance économique, de nombreux producteurs des pays pauvres restent coupés des marchés internationaux parce qu'ils ne peuvent concurrencer les biens fortement subventionnés dans les pays développés ou parce qu'ils manquent de l'infrastructure et du savoir-faire nécessaires pour participer à l'économie mondiale. L'incapacité à rompre le cercle vicieux de la pauvreté et des conflits peut avoir des conséquences régionales et mondiales. Par exemple, sur les 32 pays situés au bas de I'Indice de développement humain (IDH), 22 ont connu un conflit violent à un moment donné depuis 1990 ; et des 52 pays dans lesquels les statistiques de mortalité infantile sont constantes ou augmentent même, 30 ont connu un conflit violent depuis 1990 (BUTLER, 2011, p. 71, notre traduction) ${ }^{5}$.

\footnotetext{
5 "The poorest 40 percent of the world's population, the 2.5 billion people who live on less than $\$ 2$ a day, account for five percent of global income, while the richest 10 percent account for 54 percent. (...) Although there are technical resources and no longer the logistical obstacles to abolishing poverty, more than 800 million people suffer from hunger and malnutrition. 1 billion people do not have access to clean drinking water. While trade can be one of the most effective engines of economic growth, many producers in poor countries remain cut off from international markets because they cannot compete with heavily subsidized goods produced in the developed world or because they lack the basic infrastructure and know-how needed
} 
De cette façon, les institutions susmentionnées ont été la base de l'implémentation d'une vision spécifique de développement :

Le rôle du FMl et de la Banque mondiale est considéré comme apolitique, car il reflète les principes sans valeur mis au jour par l'économie positive (...). C'est clairement une fausse supposition à faire, car elle manifeste sa propre position politique par son programme néolibéral d'ingérence minimale du gouvernement, une économie de libre-échange, le libre-échange, la libéralisation financière et la promotion du commerce privé. Être apolitique n'est pas une option (BUTLER, 2011, p. 95 , notre traduction) ${ }^{6}$.

Il est essentiel d'affirmer que les profondes évolutions et complexifications du droit international depuis la fin de la Seconde Guerre Mondiale et plutôt à partir de la chute de l'URSS semblent avoir accentué les subtilités impérialistes, lesquelles sont plus difficiles à être perçues aujourd'hui qu'au passé. Notre argument le plus important à ce stade est que le néolibéralisme se révèle profondément impliqué dans une espèce de néocolonialisme contemporain. Une fois encore, même s'il a eu des changements de la structure et de la superstructure internationale, l'arrière-plan impérialiste semble avoir perduré à cette époque.

(...) personne ne considère plus sérieusement des alternatives possibles au capitalisme, alors que l'imagination populaire est persécutée par les visions de la " rupture de la nature ", de l'arrêt de toute vie sur terre - il semble plus facile d'imaginer « la fin du monde » qu'un changement beaucoup plus modeste dans le mode de production, comme si le capitalisme libéral était le « réel » qui survivrait d'une manière ou d'une autre même dans des conditions de catastrophe écologique mondiale. ... On peut donc affirmer catégoriquement l'existence d'une idéologie en tant que matrice générative qui régule la relation entre visible et nonvisible, entre imaginable et non-imaginable, ainsi que les changements dans cette relation (ZIZEK, 1995, p. 1, notre traduction) ${ }^{7}$.

to participate in the global economy. Failure to break the vicious circle of poverty and conflict can have regional and global consequences. For example, of the 32 countries at the bottom of the Human Development Index (HDI), 22 have experienced violent conflict at some time since 1990; and of the 52 countries in which child mortality statistics are constant or even increasing, 30 have experienced violent conflict since 1990'.

6 "The role of the IMF and the World Bank is considered apolitical, because it reflects the value-free principles uncovered by positive economics (...). This is clearly a false assumption to make, as it manifests its own political position by its neo-liberal agenda of minimal government interference, a laissez-faire free market economy, free trade, financial liberalization and the promotion of private business. To be apolitical is not an option".

7 "(...) nobody seriously considers possible alternatives to capitalism any longer, whereas popular imagination is persecuted by the visions of the forthcoming 'breakdown of nature', of the stoppage of all life on earth - it seems easier to imagine the 'end of the world' than a far more modest change in the mode of production, as if liberal capitalism is the 'real' that will somehow survive even under conditions of a global ecological catastrophe (...). One can thus categorically assert the existence of ideology qua generative matrix that regulates the relationship between visible and non-visible, between imaginable and non-imaginable, as well as the changes in this relationship". 
II semble intéressant de faire ressortir que, surtout pendant le XIXème siècle, les soi-disant traités inégaux établis ${ }^{8}$ entre une grande partie des pays du globe et les puissances mondiales (par coercition plus consensus), notamment l'Angleterre, ont approfondi la dépendance des premiers à l'encontre des deuxièmes. De cette manière, les intérêts particuliers du Royaume-Uni ont été transformés en universels, en raison de l'hégémonie presque incontestable de cet État. Le droit international, surtout à travers ce système de " traités inégaux ", semble avoir été une source indispensable pour cette hégémonie.

D'une façon similaire, gardant les proportions et les complexités appropriées, le néolibéralisme semble établir des traités inégaux au niveau planétaire, donc stipulant un type de néocolonialisme économique. Plus que jamais, le rôle de l'idéologie est fondamental pour mettre en place le néocolonialisme actuel, en raison notamment de la nécessité d'une approche moins explicite de domination.

Toutefois, non seulement le contenu, mais encore la forme du droit international se révèle potentiellement impliquée avec l'impérialisme en arrière-plan.

\section{LES SOURCES DU DROIT INTERNATIONAL ET L'IMPERIALISME}

Concernant les conflits économiques entre les États, notamment à partir de l'indépendance des ex-colonies autour du monde au 20ème siècle, dans un premier temps, les pays occidentaux affirmaient qu'ils ne seraient pas liés coutumièrement par le Nouvel Ordre Économique Internationale ${ }^{9}$, en raison de la possibilité d'une objection persistante ; de surcroît, et paradoxalement, des nouveaux États avaient besoin de respecter les anciennes règles économiques, étant donné que ces règles étaient une des conditions pour l'acceptation de l'indépendance des nouvelles nations (ANGHIE, 2006).

Le droit international d'aujourd'hui semble chercher l'établissement des lois internationales dans les États dépendants et dominés, à partir d'une libéralisation au-delà des contrôles aux frontières et de la réorganisation des compétences des États (CUTLER, 2008). Un exemple intéressant de cette affirmation se traduit dans la séparation doctrinale et jurisprudentielle des actes

\footnotetext{
${ }^{8}$ Les soi-disant Traités Inégaux ont été une série de traités signés notamment au XIXème siècle entre les pays subordonnés et les puissances occidentales industrialisées. Ces traités ont établi des profondes concessions économiques auxdits puissances par ces États dominés, notamment des bas tarifs douaniers, sans toutefois obtenir de réciprocité (SARAIVA, 2007).

${ }^{9}$ Le nouvel ordre économique international est une notion impulsée dans les années 1960 par les pays du tiers monde pour exprimer leurs revendications dans le domaine des relations commerciales, de sorte que les États les plus fragiles puissent bénéficier d'avantages spécifiques par rapport aux États déjà développés.
} 
jure imperium et des actes jure gestionis, par laquelle s'étend la compétence des juridictions internationales sur les actes commerciaux internationaux des États tiers.

Dans les domaines clés de la vie économique, sociale et politique souveraine, les États dominés ne peuvent pas prendre des décisions indépendantes, car ceux-ci ont cédés ses pouvoirs au droit international et aux institutions (CHIMNI, 2008, p. 60 , notre traduction) ${ }^{10}$.

De ce fait, les intérêts dominants sont médiatisés par ces institutions, afin d'apporter des solutions légitimes aux yeux des populations à l'échelle mondiale et de codifier les intérêts des classes dominantes. Donc, ces efforts en vue d'établir des lois internationales et institutionnelles qui facilitent la mondialisation de la production sont indispensables pour la cristallisation des pouvoirs des grandes puissances sur les États et peuples subalternes. Selon Chimni (2008), la prescription de la « gouvernance démocratique » offre des États capitalistes avancés un prétexte pour intervenir contre des forces qui ne favorisent pas leur économie et leur intérêts géostratégiques, pour autant qu'il puisse être établi que ces forces sont en violation des normes libérales-démocratiques.

Étant donné l'identification entre le droit international et les intérêts des grandes puissances, la violation dudit droit n'est pas un événement fréquent. Nonobstant, compte tenu des changements et des relations dialectiques entre ces intérêts et ce droit, il est difficile d'avoir une identification homogène entre ceux-ci (BUTLER, 2011; CHIMNI, 2008; ORFORD, 2003). Par conséquent, il existe toujours un décalage. Afin de résoudre ladite querelle, deux possibilités sont possibles : la transformation des règles (comme l'institution de l'OMC dans les années 1990's) ou des violations de celles-ci (l'usage de la force dans des situations non-prévues par la Charte des Nations Unis) (CHIMNI, 2008).

Dans ce contexte, étant donné que le processus politique mondial fait prévaloir des mouvements de négociation et de coercition plutôt que de persuasion, les sources du droit international sont aussi impliquées dans la réplication des structures de forces (MIÈVILLE, 2006, 2008). S'agissant des traités, Chimni (2008) assure que certains intérêts des classes subalternes ne sont pas pris en compte pendant les négociations internationales, au vu de l'inexistence d'une démocratie participative au sein de celles-ci. De surcroît, chaque accord international est l'expression d'un ordre social établi avec un certain équilibre. Par conséquent, une obligation conventionnelle n'est rien d'autre qu'une concrétisation des relations économiques et politiques, c'est-à-dire que la réalité extratextuelle des accords est minimisée.

\footnotetext{
10 "In key areas of sovereign economic, social and political life, the dependent and dominated state cannot
} take independent decisions, since it has ceded its powers to international law and institutions". 
Néanmoins, aucune source n'est plus influencée par les intérêts dominants que le droit coutumier, sous prétexte que cette source manifeste étroitement la volonté des États puissants ; en ce sens, la formation de /'opinio juris ${ }^{11}$ est directement dépendante des opinions des grandes puissances (CHIMNI, 2008; PASHUKANIS, 1978). Concernant, par exemple, la possession et l'utilisation d'armes nucléaires, bien que l'ensemble de la société internationale révèlent être d'accord quant à l'illégalité de l'utilisation et de la prolifération de ce types d'armes, la formation d'une norme coutumière semble impossible dans ce contexte, car cette situation serait complètement contre les intérêts des pays qui possèdent des armes nucléaires (ceux-ci correspondent globalement aux grandes puissances économiques et politiques mondiales actuelles, sauf quelques exceptions) (CHIMNI, 2008).

De plus, la conception de l'objecteur persistant s'adapte facilement aux intérêts dominants, empêchant l'application d'une norme coutumière contraire à ces intérêts. En conséquence, au vu de ce mode de législation plus souple, il semble difficile pour cette source de devenir un instrument de changement pour les États et les classes subalternes. En outre, toujours conformément à Chimni (2008), ceci met en lumière la raison selon laquelle le droit international n'accepte pas des sources plus démocratique, compte tenu de ces caractéristique plus « douces » (la soft law).

Accepter de nouvelles sources démocratiques signifierait la restructuration radicale du système international au détriment de la classe capitaliste internationale. En d'autres termes, alors que la "soft law » reflète d'intérêts généralisables, la « hard law », dans un ordre mondial bourgeois, incarne d'intérêts particuliers. Sans surprise, les idéaux de la démocratie délibérative et de la justice distributive qui informe beaucoup "soft law " sont confinés aux processus politiques au sein des États-nations, permettant à l'impérialisme de s'échapper à la responsabilité de l'état transnational (CHIMNI, 2008, p. 72, notre traduction) ${ }^{12}$.

De ce fait, lesdites sources sont des produits plus souvent d'une action communicative ; par conséquent, celles-ci sont marginalisées par le système juridique international. Enfin, alors que le conflit est une réalité constante, le droit international et son interprétation positiviste met en évidence une universalité et un consensus inexistants dans le monde matériel. Selon Kennedy (1987), le discours des sources semble prendre la qualité d'une essence désincarnée. Nonobstant, toutes les vérités sont construites à travers des relations intersubjectives, lesquelles sont radicalement instables. En ce sens, l'objectivité et le consensus ne semblent pas possibles.

\footnotetext{
${ }^{11}$ L'opinio juris est l'opinion selon laquelle une action a été menée comme une obligation légale. Celui-ci constitue l'élément psychologique essentiel à la formation de la coutume internationale.

12 "(...) to accept new democratic sources would mean the radical restructuring of the international system to the disadvantage of the international capitalist class. To put it differently, while 'soft law' reflects generalizable interests, hard law in a bourgeois world order embodies particular interests. Unsurprisingly, the ideals of deliberative democracy and distributive justice that inform much 'soft law' are confined to political processes within nation-states, allowing imperialism to escape transnational state responsibility".
} 
Par conséquent, il semble que le formalisme et le contenu actuel du droit international soient des formes implicites d'exclusion, du fait que le droit susmentionné est une partie d'une superstructure et d'un projet bien défini (CARTY, 1991; 2008). L'abstraction et l'objectivité du droit semble servir aux intérêts de ceux qui cherchent à cacher les intérêts idéologiques derrière ce contexte. Enfin, le discours juridique international est enraciné dans une pratique et une tradition de droit préétablie.

\section{CONCLUSION}

Dans ce contexte, il est crucial de souligner que le néolibéralisme semble approfondir non seulement les divisions sociales, mais aussi valide et rend légitime ces inégalités. Selon la conclusion de l'article "Polany's « double movement": The belle époque of British and US hegemony compared», de Beverly Silver et Giovanni Arrighi (2003, p. 349, notre traduction) ${ }^{13}$, «le vrai problème est que certains pays ont le pouvoir de faire fonctionner le marché mondial à leur avantage, tandis que d'autres n'ont pas ce pouvoir et doivent supporter les coûts ».

II est nécessaire ici d'assurer l'importance de cette explication sur l'hégémonie mondial néolibéral, dans la mesure où le droit international est une partie de cette superstructure bourgeoise. En conséquence, le droit économique et la Gouvernance Économique Internationale souffrent de ces mêmes influences idéologiques déterminantes.

L'État postcolonial a, à bien des égards, adopté les modèles de développement, de progrès et d'État-nation qui avaient d'abord été articulés dans le système mandataire et qui avaient été affinés et élaborés par des théories du développement telles que la théorie de la modernisation (ANGHIE, 2006, p. 749, notre traduction) ${ }^{14}$.

De ceci, à partir des sources et des arguments susmentionnés, le colonialisme formel semble avoir été remplacé vers les années 1970 par un type de néocolonialisme économique, étant donné que les dépendances économiques sont imposées aux États subalternes, notamment par les institutions internationales en utilisant le droit international et l'idéologie dominante en arrièreplan de celui-ci. Dans ce contexte, le néolibéralisme pourrait être compris comme un « traité inégal à l'échelle mondiale ".

\footnotetext{
13 "The real problem is that some countries have the power to make the world market work to their advantage, while others do not have that power and have to bear the costs".

14 "The postcolonial state, in many ways, adopted the models of development, progress and the nation-state that had first been articulated in the Mandate System and that had been further refined and elaborated by development theories such as modernization theory".
} 
* Artigo recebido em 23 de agosto de 2019, aprovado em 07 de novembro de 2019.

\section{BIBLIOGRAPHIE}

ANGHIE, A. The evolution of international law: colonial and postcolonial realities. Third World Quaterly, v. 27, n. 5, p. 739-753, 2006.

ARRIGHI, G.; SILVER, B. J. Polanyi's "double movement": the belle époque of British and US hegemony compared. Politics \& Society, v. 31, n. 2, p. 325-355, 2003.

BIANCHI, A. International law theories: an inquiry into different ways of thinking. Oxford: Oxford University Press, 2016.

BM. Accord de la Banque Mondiale, 1989. Disponível em: <http://siteresources.worldbank.org/EXTABOUTUS/Resources/ibrd-articlesofagreement.pdf>

BUTLER, K. A critical humanitarian intervention approach. Warwich: Department of politics and international studies, 2011.

CARTY., A. Critical international law: recent trends in the theory of international law. European Journal of International Law, v. 2, n. 1, p. 66-96, 1991.

CARTY, A. Marxism and international law: perspectives for the American (twenty-first) century? In: MARKS, S. (Ed.). International law on the left: re-examining Marxist legacies. Cambridge: Cambridge University Press, 2008. p. 169-198.

CHIMNI, B. S. An outline of a Marxist course on public international law. In: MARKS, S. (Ed.). International law on the left: re-examining Marxist legacies. Cambridge: Cambridge University Press, 2008. p. 53-91.

COX, R. Social forces, states and world orders: beyond International relations theory. Millennium: journal of international studies, v. 10, n. 2, p. 126-155, 1981.

COX, R.; SCHECHTER, M. G. The political economy of a plural world: Critical reflections on power, morals and civilization. London and New York: Routledge/RIPE, 2002.

CUTLER, A. C. Toward a radical political economy critique of transnational economic law. In: MARKS, S. (Ed.). International law on the left: re-examining Marxist legacies. Cambridge: Cambridge University Press, 2008. p. 199-219.

FILHO, P. P. O ajustamento na América Latina: crítica ao modelo de Washington. Lua Nova, n. 32, p. 101-131, 1994.

FMI. Statuts du Fond Monétaire International, juillet 1944. Disponível em: <https://www.imf.org/external/french/pubs/ft/aa/aa.pdf> 
FMI. IMF Members' Quotas and Voting Power, and IMF Board of Governors, 2018. Disponível em: <http://www.imf.org/external/np/sec/memdir/members.aspx>

GILPIN, R. U.S. power and the multinational corporation: the Political Economy of Foreign Direct Investment. New York: Basic Books, 1975.

GILPIN, R. Global Political Economy. Understanding the International Economic Order. Princeton: Princeton University Press, 2001.

GRAMSCI, A. Os Intelectuais e a Organização da Cultura. Rio de Janeiro: Civilização Brasileira, 1982.

HIRSHC, M. L'École de Francfort: une critique de la raison instrumentale. L'Homme et la société, Marxisme critique et idéologie. n. n. 35-36, p. 115-147, 1975.

KENNEDY, D. International legal structure. Baden-Baden: Nomos Verlagsgesellschaft, 1987.

KEOHANE, R.; NYE, J. Power and interdependence: world politics in transition. New York: Longman, 2001.

KOSKENIEMI, M. What should international lawyers learn from Karl Marx? In: MARKS, S. (Ed.). International law on the left: re-examining Marxist legacies. Cambridge: Cambridge University Press, 2008. p. 30-52.

KRASNER, S. State power and the structure of international trade. World Politics, v. 28, n. 3, p. 317-347, 1976.

MARKS, S. Exploitation as an international legal concept. In: MARKS, S. (Ed.). International law on the left: re-examining Marxist legacies. Cambridge: Cambridge University Press, 2008. p. 281-308.

MARX, K. Grundrisse. Manuscritos econômicos de 1857-1858: Esboços da crítica da economia política. São Paulo: Boitempo, 2011.

MARX, K.; ENGELS, F. A ideologia alemã: crítica da mais recente filosofia alemã em seus representantes Feuerbach, B. Bauer e Stirner, e do socialismo alemão em seus diferentes profetas (1845-1846). São Paulo: Hucitec, 1999.

MIÈVILLE, C. Between equal rights: a Marxist theory of international law. Chicago: Haymarket Books, 2006.

MIÈVILLE, C. The commodity-form theory of international law. In: MARKS, S. (Ed.). International law on the left: re-examining Marxist legacies. Cambridge: Cambridge University Press, 2008. p. 92132.

MORTON, A. D. Unravelling Gramsci: Hegemony and Passive Revolution in the Global Political Economy. London: Pluto Press, 2007.

OLIVEIRA, G.; MAIA, G.; MARIANO, J. O sistema de Bretton Woods e a dinâmica do sistema monetário internacional contemporâneo. Pesquisa \& Debate, v. 19, n. 2 (34), p. 195-2019, 2008.

ORFORD, A. Reading humanitarian intervention: human rights and the use of force in international law. Cambridge: Cambridge University Press, 2003.

PAQUIN, S. Économie Politique Internationale. Paris: Montchrestien, 2009. 
PASHUKANIS, E. Law and Marxism: a general theory. London: InkLinks, 1978.

PIJL, K. V. D. Transnational classes and international relations. London and New York: Routledge, 2005.

POLANYI, K. A grande transformação: as origens de nossa época. $2^{a}$ ed. Rio de Janeiro: Campus, 2000.

RUPERT, M. Alienação, capitalismo e o sistema interestados. In: GILL, S. (Ed.). Gramsci, materialismo histórico e relações internacionais. Rio de Janeiro: Editora UFRJ, 2007.

SARAIVA, J. F. DE S. História das Relações Internacionais Contemporâneas: Da sociedade internacional do século XIX à era da globalização. $2^{a}$ ed. São Paulo: Saraiva, 2007.

SILVA, E. Exchange rising? Karl Polanyi and contentious politics in contemporary Latin America. Latin American Politics and Society, v. 54, n. 3, p. 1-32, 2012.

STRANGE, S. The retreat of the State: the diffusion of power in the World Economy. Cambridge: Cambridge University Press, 1996.

STRANGE, S. Casino capitalism. Manchester: Manchester University Press, 1997.

THOMAS, C. Global governance, development and human security. London: Pluto Press, 2003.

VIGEVANI, T. et al. A contribuição marxista para o estudo das Relações Internacionais. Lua Nova, n. 83, p. 111-143, 2011.

ZIZEK, S. Mapping ideology. London/New York: New Left Books, 1995. 